Surface Review and Letters, Vol. 9, No. 2 (2002) 1251-1255

(C) World Scientific Publishing Company

\title{
EXPERIMENTAL AND THEORETICAL STUDIES OF THE ELECTRONIC STRUCTURES OF $\mathrm{AuAl}_{2}$ AND PtGa
}

\author{
L.-S. HSU \\ Department of Physics, National Chang-Hua University of Education, \\ Chang-Hua 500, Taiwan, ROC \\ G. Y. GUO \\ Department of Physics, National Taiwan University, \\ Taipei 106, Taiwan, ROC \\ J. D. DENLINGER and J. W. ALLEN \\ Randall Laboratory, University of Michigan, Ann Arbor, \\ Michigan 48109-1120, USA
}

\begin{abstract}
Synchrotron-radiation-excited angle-resolved photoemission spectra for the (100) and (111) faces of $\mathrm{AuAl}_{2}$ and the (111) face of $\mathrm{PtGa}_{2}$ are presented, along with derived experimental dispersion relations. These bands are compared with those obtained from the first-principles theoretical band-structure calculations. The total densities of states at the Fermi energy for these two materials are compared with those obtained from the specific-heat measurements. The energy variations of $\mathrm{Au}$ and $\mathrm{Pt} 5 \mathrm{~d}$ bands for $\mathrm{AuAl}_{2}$ and $\mathrm{PtGa}_{2}$, respectively, show atomic-like behavior in the photon-energy range of 80-220 eV.
\end{abstract}

\section{Introduction}

$\mathrm{AuAl}_{2}$ and $\mathrm{PtGa}_{2}$ are intermetallic compounds of both technological and scientific importance. The former has potential applications as a selective solar absorber, ${ }^{1}$ and the latter was proposed to be used as a thermodynamically stable conducting contact to GaAs. ${ }^{2}$ They both crystallize in the cubic fluorite structure, and superconduct at low temperatures. ${ }^{3}$ They are also prototype materials for studying the $\mathrm{Au}$ and $\mathrm{Pt} 5 \mathrm{~d}$ bands in intermetallic compounds, since group-III metals contribute only $\mathrm{s}-\mathrm{p}$ states to the valence band (VB). The purple and gold colors of $\mathrm{AuAl}_{2}$ and $\mathrm{PtGa}_{2}$, respectively, also strongly motivated researchers to study the electronic structures of these two materials. Many electronic and physical properties of these two compounds have been measured, and a majority of these works were reviewed by Hsu. ${ }^{3}$ This paper will focus on angle-resolved photoemission-spectroscopic (ARPES) study and theoretical band-structure calculation of $\mathrm{AuAl}_{2}$ and $\mathrm{PtGa}_{2}$.

\section{Experiment and Theory}

Procedures for preparation of the (100) and (111) faces of $\mathrm{AuAl}_{2}$ and the (111) face of $\mathrm{PtGa}_{2}$ single crystals were the same as those reported previously. ${ }^{4,5}$ The ARPES experiments were performed at the Advanced Light Source. Photon energies in the range of $80-220 \mathrm{eV}$ were selected with a spherical-grating monochromator, and a Physical Electronics 137-mm hemispherical analyzer was used in the fixed-analyzer-transmission mode to collect the photoemission spectra. With 150 lines/mm for grating setting and $3 \mathrm{eV}$ for analyzer pass-energy setting, the energy resolution was less than $80 \mathrm{meV}$. The angular resolution was less than $\pm 1^{\circ}$, which corresponds to a momentum resolution of roughly $0.2 \AA^{-1}$ at $125 \mathrm{eV}$ kinetic energy. The vacuum chamber was also equipped with a double-anode ( $\mathrm{Al}$ and $\mathrm{Mg}$ ) X-ray source for X-ray photoemission spectroscopy (XPS) and X-ray photoelectron diffraction (XPD) measurements, and with a low-energy electron diffraction (LEED) system. The chamber 
pressure during the experiments was $2 \times 10^{-10}$ Torr. The samples were cleaned by repeated cycles of sputtering with Ar ions and annealing to $500^{\circ} \mathrm{C}$ until XPS spectra showed no traces of $\mathrm{O}$ or $\mathrm{C}$ contamination on the sample surfaces. The observed XPD and LEED patterns ${ }^{6}$ also confirmed the cleanliness and ordering of the sample surfaces.

To interpret the ARPES data, we have calculated the relativistic band structures of $\mathrm{AuAl}_{2}$ and $\mathrm{PtGa}_{2}$ with the $\mathrm{CaF}_{2}$ structure and at the experimental lattice constants of 5.99 and $5.91 \AA$, respectively. We used the highly accurate all-electron full-potential linear augmented-plane-wave (FLAPW) method. ${ }^{7}$ The calculations are based on the first-principles density functional theory with the standard local density approximation to the exchange-correlation potential. $^{8}$ The muffin-tin radii used for the constituent atoms of $\mathrm{AuAl}_{2}$ and $\mathrm{PtGa}_{2}$ are $1.27 \AA$. The shallow $5 \mathrm{p}$ and $4 \mathrm{f}$ core states of $\mathrm{Au}$ and $\mathrm{Pt}$ and the $3 \mathrm{~d}$ states of $\mathrm{Ga}$ were also treated as band states by using the so-called local orbitals. ${ }^{7}$ The wave functions, the charge densities, and the potentials are expanded in terms of the spherical harmonics inside the muffin-tin spheres. The cutoff angular momentum $\left(l_{\max }\right)$ is 10 for the wave functions and six for the charge densities and the potentials. The Brillouin zone (BZ) integration is carried out by using the improved tetrahedron method. ${ }^{9}$ The number of the augmented plane waves included is about 120 per atom, i.e. $R_{\mathrm{mt}} K_{\max }=9 .^{7}$ The number of the $\mathrm{k}$ points in the irreducible BZ wedge (IBZW) used in the self-consistent calculations is 72 . To calculate the total density of states $(\mathrm{DOS})$ at the Fermi energy $\left(E_{\mathrm{F}}\right), 165 \mathrm{k}$ points in the IBZW were used. We have checked the convergence of the calculated eigenvalues with respect to the number of augmented plane waves used and the number of $\mathrm{k}$ points used. In particular, we found that increasing $R_{\mathrm{mt}} K_{\max }=9$ to 10 changed the relative positions of the eigenvalues at the $\Gamma$ points by up to a couple of meV only.

\section{Results and Discussion}

Figure 1 shows a set of VB photoemission spectra from the (100) and (111) faces of $\mathrm{AuAl}_{2}$, and from the (111) face of $\mathrm{PtGa}_{2}$ at normal emission. The normals to the sample surfaces were determined from the $\mathrm{Au}$ and $\mathrm{Pt} 4 \mathrm{f}_{7 / 2}$ XPD polar and azimuth scans. ${ }^{6}$ The photon energy $(h \nu)$ was varied so as to probe the third to the fourth BZ's for the (100) face,
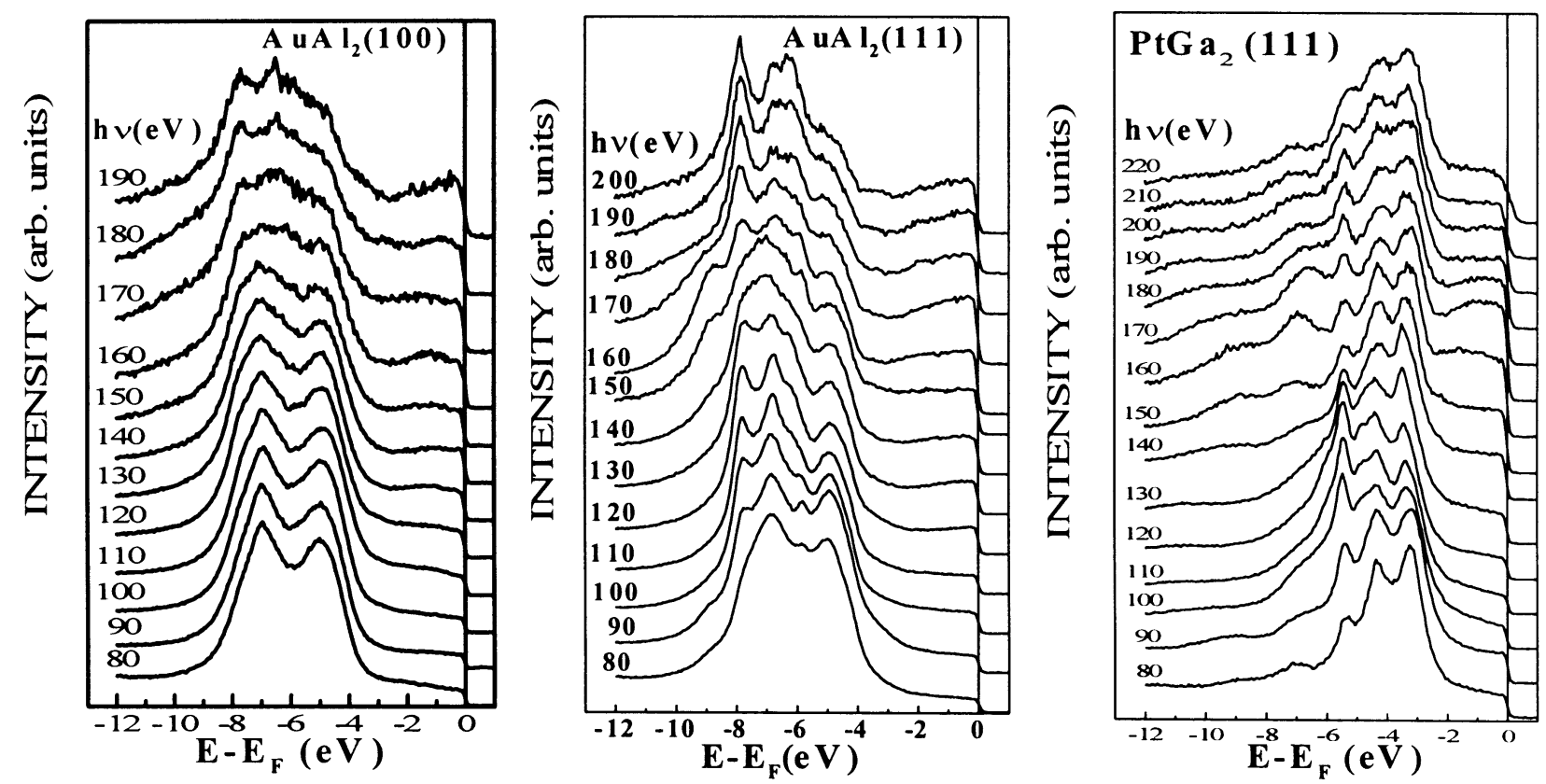

Fig. 1. Angle-resolved photoemission spectra taken at normal emissions from the (100) and (111) faces of $\mathrm{AuAl}_{2}$, and the (111) face of $\mathrm{PtGa}_{2}$. 
and the fourth to fifth BZ's for the (111) face of these two materials. The $E_{\mathrm{F}}$ was assigned at half height on the onset of a plateau, which is caused by photoemission from the Au or Pt s-p band. In general, s-p bands disperse much more rapidly than the $\mathrm{d}$ bands, thus accounting for the broader features seen in the photoemission spectra. At $h \nu \geq 160 \mathrm{eV}$, more final states are accessible for the $\mathrm{Au}$ and $\mathrm{Pt}$ s-p states, which causes the higher intensity observed in the VB spectra. In Fig. 1, a three-peak structure with much higher intensity at higher binding energy $\left(E_{\mathrm{B}}\right)$ is due to the $\mathrm{Au}$ or Pt $5 \mathrm{~d}$ states. The $E_{\mathrm{B}}$ 's of these peaks change less than $0.3 \mathrm{eV}$ by varying $h \nu$, and are listed in the first three columns of Table 1 . We note that the $E_{\mathrm{B}}$ values at the three $\Gamma$ points (at approximately $h \nu=110$ or $220 \mathrm{eV}$ ) determined form the present ARPES data for $\mathrm{PtGa}_{2}$ are 3.40, 4.35, and $5.45 \mathrm{eV}$, which are essentially the same as those obtained from the total DOS features in the angle-integrated photoemission spectra. ${ }^{4}$ This means that the Pt $5 \mathrm{~d}$ bands in $\mathrm{PtGa}_{2}$ are well localized. We should also point out that these $E_{\mathrm{B}}$ values are within $10 \%$ of the corresponding values $(3.55,4.45$, and $5.90 \mathrm{eV})$ for elemental $\mathrm{Au},{ }^{10}$ which explains why $\mathrm{PtGa}_{2}$ is gold-colored.

By using a direct-transition model and assuming free-electron conduction-band structures, the energy-versus-momentum dispersion relations for $\mathrm{AuAl}_{2}$ along $\mathrm{X}-\Gamma-\mathrm{L}$ and for $\mathrm{PtGa}_{2}$ along $\mathrm{L}-\mathrm{\Gamma}-\mathrm{L}$ are plotted in Figs. 2 and 3, respectively, as open circles. The values of the inner potentials used for $\mathrm{AuAl}_{2}$ and $\mathrm{PtGa}_{2}$ were estimated to be 12.0 and $12.3 \mathrm{eV}$, respectively. These values were taken to be the differences between the muffin-tin zeros of energy in

Table 1. The experimental (ARPES work, first line) and theoretical (FLAPW work, second line) binding energies $\left(E_{\mathrm{B}}\right)$ of the $\mathrm{Au}$ and $\mathrm{Pt} 5 \mathrm{~d}$ states at $\Gamma$, the crystal field parameters (10Dq), and the spin-orbit parameters $(\xi)$ for $\mathrm{Au}$ metal, $\mathrm{AuAl}_{2}$ and $\mathrm{PtGa}_{2}$. All values in $\mathrm{eV}$.

\begin{tabular}{lccccc}
\hline \hline Material & $E_{\mathrm{B}}\left(\Gamma_{8}^{1}\right)$ & $E_{\mathrm{B}}\left(\Gamma_{7}\right)$ & $E_{\mathrm{B}}\left(\Gamma_{8}^{2}\right)$ & $10 \mathrm{Dq}$ & $\xi$ \\
\hline $\mathrm{Au}$ & 3.55 & 4.45 & 5.90 & 1.22 & 0.71 \\
& 3.09 & 4.34 & 5.52 & 1.60 & 0.61 \\
$\mathrm{AuAl}_{2}$ & 4.90 & 5.80 & 6.85 & 1.18 & 0.53 \\
& 5.47 & 6.23 & 7.25 & 1.02 & 0.51 \\
$\mathrm{PtGa}_{2}$ & 3.40 & 4.40 & 5.47 & 0.9 & 0.56 \\
& 3.34 & 4.88 & 5.45 & 1.80 & 0.33 \\
\hline \hline
\end{tabular}

the present FLAPW calculation $(7.4$ and $7.7 \mathrm{eV}$ ) and the vacuum levels as determined from the work function $(4.63 \mathrm{eV})^{11}$ of $\mathrm{AuGa}_{2}$. The band structures calculated by the FLAPW method for $\mathrm{AuAl}_{2}$ along $\Delta$ and $\Lambda$ and for $\mathrm{PtGa}_{2}$ along $\Lambda$ are plotted as closed circles in Figs. 2 and 3, respectively. The

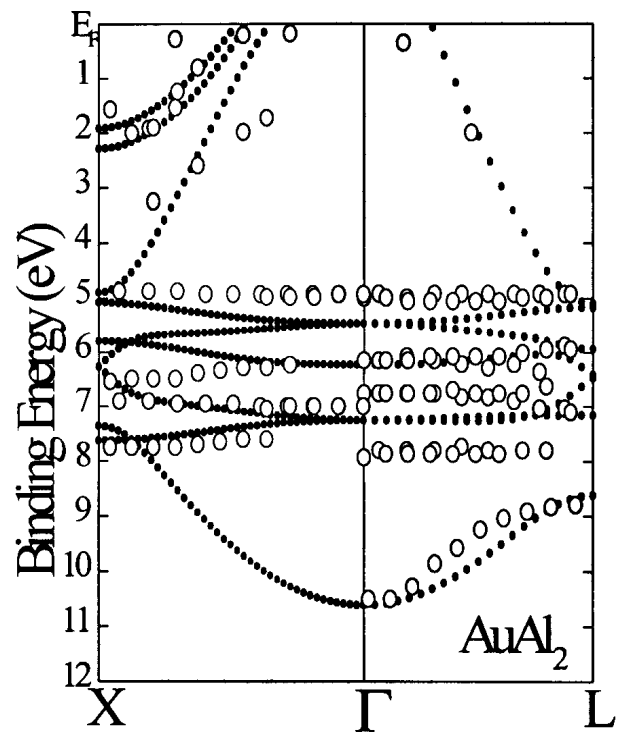

Fig. 2. Band structure of $\mathrm{AuAl}_{2}$ along $\mathrm{X}-\mathrm{\Gamma}-\mathrm{L}$. Open circles are bands derived from the ARPES data. Closed circles are theoretical band structure calculated by the FLAPW method.

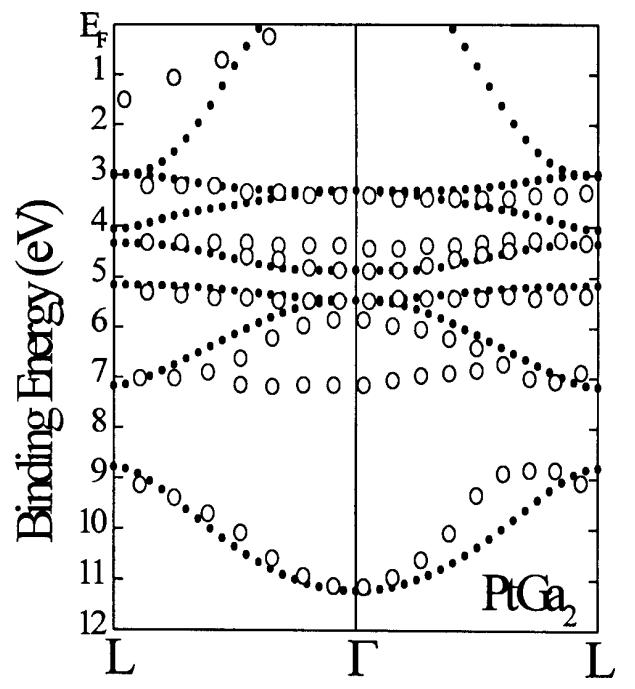

Fig. 3. Band structure of $\mathrm{PtGa}_{2}$ along $\mathrm{L}-\Gamma-\mathrm{L}$. Open circles are bands derived from the ARPES data. Closed circles are theoretical band structure calculated by the FLAPW method. 
agreement between the experimental and theoretical bands is rather good for both compounds, which indicates that the spin-orbit effect is important in calculating the band structures of these materials. The $E_{\mathrm{B}}$ values at $\Gamma$ points determined form present FLAPW calculation for $\mathrm{Au}, \mathrm{AuAl}_{2}$, and $\mathrm{PtGa}_{2}$ are listed under the corresponding ARPES values in Table 1 . The crystal-field and the spin-orbit parameters of $\mathrm{AuAl}_{2}$ and $\mathrm{PtGa}_{2}$ were determined in the same manner as was done previously ${ }^{4}$ and are listed in the last two columns of Table 1. The experimental band for $\mathrm{PtGa}_{2}$ around $E_{\mathrm{B}}=7 \mathrm{eV}$ is due to emissions from the one-dimensional DOS. ${ }^{12}$ It is identified as such because it resonates strongly at $h \nu=160$ and $170 \mathrm{eV}$, at which energies the BZ boundary is reached; and is rather weak between $h \nu=110$ and $130 \mathrm{eV}$, at which energies the center of the $\mathrm{BZ}$ is covered. The experimental $\mathrm{PtGa}_{2}$ band at $E_{\mathrm{B}}=$ $4.7 \mathrm{eV}$ for $h \nu=100-130 \mathrm{eV}$ involves a surface state. This surface state was checked by exposing the sample surface with oxygen and observing its gradual disappearance.

The DOS at $E_{\mathrm{F}}\left[n\left(E_{\mathrm{F}}\right)\right]$ values calculated by the FLAPW method for $\mathrm{AuAl}_{2}$ and $\mathrm{PtGa}_{2}$ are 0.92 and 1.15 states/eV-cell, respectively. These values are comparable to those $\left(1.08^{13}\right.$ and $1.09^{14}$ states $/ \mathrm{eV}$ cell, respectively) calculated by using the mixedbasis band-structure interpolation scheme method. The $n\left(E_{\mathrm{F}}\right)$ values for $\mathrm{AuAl}_{2}$ and $\mathrm{PtGa}_{2}$ obtained from the specific-heat measurements $\left(1.28^{15}\right.$ and $1.67^{16}$ states/eV-cell, respectively) are these "bandstructure" DOS enhanced by a factor $1+\lambda$, where $\lambda$ is the electron-photon mass enhancement parameter. The $\lambda$ values thus determined for $\mathrm{AuAl}_{2}$ and $\mathrm{PtGa}_{2}$ are 0.39 and 0.45 , respectively. We note that the $\lambda$ value for $\mathrm{AuAl}_{2}$ is $34 \%$ larger than the value $(0.29)$ calculated by using McMillan's formula ${ }^{17}$ with critical temperature $\left(T_{\mathrm{c}}\right)$ of $0.095 \mathrm{~K}$ and Debye temperature $\left(\theta_{\mathrm{D}}\right)$ of $297 \mathrm{~K} .{ }^{18}$ In comparison, the $\lambda$ value for $\mathrm{PtGa}_{2}$ is within $8 \%$ of the value $(0.49)$ calculated by using the same formula with $T_{\mathrm{c}}=2.15 \mathrm{~K}$ and $\theta_{\mathrm{D}}=220 \mathrm{~K} .{ }^{16}$ The photon-energy dependence of the $\mathrm{Au}$ and $\mathrm{Pt} 5 \mathrm{~d}$-band intensities for $\mathrm{AuAl}_{2}$ and $\mathrm{PtGa}_{2}$ in the range $80 \leqslant h \nu \leqslant 220 \mathrm{eV}$ are shown in Fig. 4 as filled squares and crosses. Also shown in Fig. 4 as solid lines are the atomic cross sections of the $\mathrm{Au}$ and Pt $5 \mathrm{~d}$ states calculated by Yeh and Lindau ${ }^{19}$ for elemental $\mathrm{Au}$ and Pt. The data points were obtained by correcting the photoemission spectra for a Shirleytype inelastic background, ${ }^{20}$ measuring the areas under the photoemission energy distribution curves in the range $3.5 \leqslant E_{\mathrm{B}} \leqslant 9.0 \mathrm{eV}$ and $2 \leqslant E_{\mathrm{B}} \leqslant 6.5 \mathrm{eV}$ for $\mathrm{AuAl}_{2}$ and $\mathrm{PtGa}_{2}$, respectively, and correcting these areas with respect to the incident photon flux. They were then normalized to the atomic crosssection values at $h \nu=80$ and $200 \mathrm{eV}$ for $\mathrm{AuAl}_{2}$ and $\mathrm{PtGa}_{2}$, respectively. The bump in the experimental data for $\mathrm{PtGa}_{2}$ in the range $h \nu=100-140 \mathrm{eV}$ is caused by non-d-weight bands dispersing outside of the energy window, $2-6.5 \mathrm{eV}$, for area integration. Namely, the sharp peak at $E_{\mathrm{B}}=5.5 \mathrm{eV}$ for $\mathrm{PtGa}_{2}$ in Fig. 1 exists precisely in this range of $100-140 \mathrm{eV}$, which corresponds to the top of the lower Pt $5 \mathrm{~d}$ band. This lower $5 \mathrm{~d}$ band disperses to below $7 \mathrm{eV}$ along
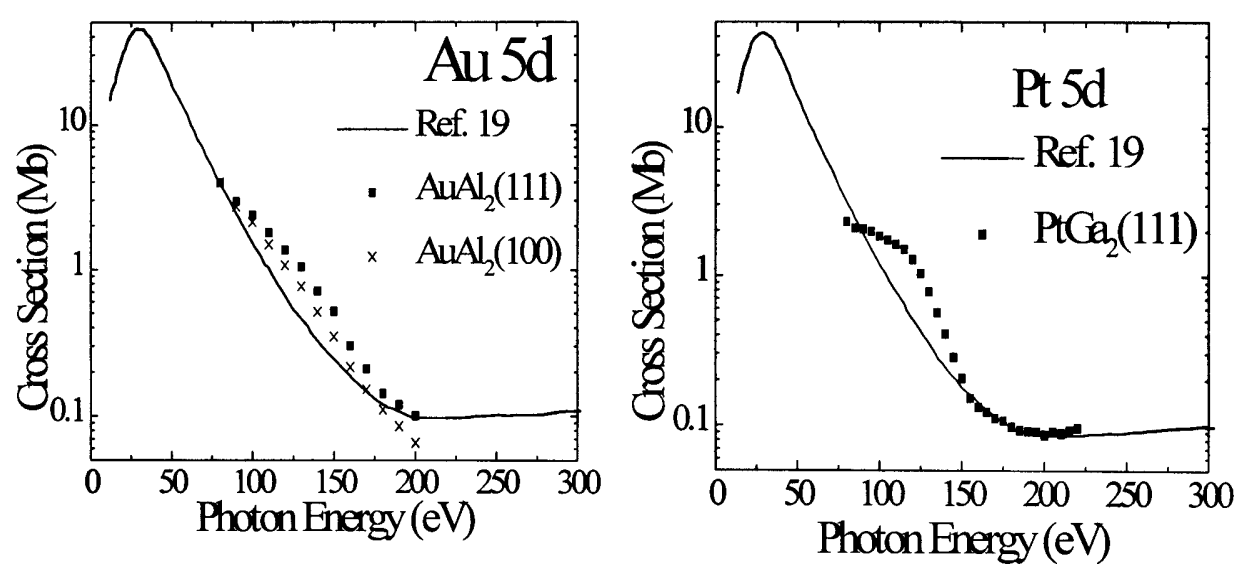

Fig. 4. Photon-energy dependence of the $\mathrm{Au}$ and $\mathrm{Pt} 5 \mathrm{~d}$-band intensities for $\mathrm{AuAl}_{2}$ and $\mathrm{PtGa}_{2}$, respectively. The solid lines are the atomic cross sections ${ }^{19}$ of the $\mathrm{Au}$ and $\mathrm{Pt} 5 \mathrm{~d}$ states for elemental $\mathrm{Au}$ and $\mathrm{Pt}$, respectively. 
$\Gamma-\mathrm{L}$ and mixes more with the s-p bands than the other two 5d bands do (the present FLAPW calculation shows that the $\mathrm{Pt} d$ weight for $\mathrm{PtGa}_{2}$ is $96 \%$, $43 \%$, and $48 \%$ at the $\Gamma_{8}^{1}, \Gamma_{7}$, and $\Gamma_{8}^{2}$ points, respectively). We note that in this photon-energy range, the $\mathrm{Au}$ and $\mathrm{Pt} 5 \mathrm{~d}$ states of $\mathrm{AuAl}_{2}$ and $\mathrm{PtGa}_{2}$, respectively, show atomic-like behavior, which is in sharp contrast to the solid-state effect observed for the $\mathrm{Au}$ and $\mathrm{Pt} 5 \mathrm{~d}$ states in $\mathrm{Au}$ and Pt metals. ${ }^{21,22}$

\section{Acknowledgments}

This work was supported by the National Science Council, Taiwan, ROC, Grants NSC89-2112-M018019 (LSH) and NSC89-2112-M002-0025 (GYG), the US Department of Energy (Grant No. DE-FG02-90ER45416), and the US National Science Foundation (Grant No. DMR-94-23741).

\section{References}

1. R. E. Hahn and B. O. Seraphin, Phys. Thin Films 10, 1 (1978)

2. Y. K. Kim, D. A. Baugh, D. K. Shuh, R. S. Williams, L. P. Sadwick and K. L. Wang, J. Mater. Res. 5, 2139 (1990)

3. L.-S. Hsu, Mod. Phys. Lett. B8, 1297 (1994), and references therein.

4. L.-S. Hsu and K.-L. Tsang, Phys. Rev. B56, 6615 (1997)

5. L.-S. Hsu, H.-W. Huang and K.-L. Tsang, J. Phys. Chem. Solids 59, 1205 (1998).

6. L.-S. Hsu, J. D. Denlinger and J. W. Allen, in Applications of Synchrotron Radiation Techniques to
Materials Science $I V$, eds. S. M. Mini, D. L. Perry, S. R. Stock and L. J. Terminello, MRS Symposia Proceedings No. 524 (Materials Research Society, Pittsburgh, 1998), p. 179.

7. P. Blaha, K. Schwarz and J. Luitz, computer code WIEN97 (Vienna University of Technology, 1997). [Improved and updated UNIX version of the original copyrighted WIEN code, which was published by P. Blaha, K. Schwarz, P. Sorantin and S. B. Trickey, Comput. Phys. Commun. 59, 399 (1990).]

8. S. H. Vosko, L. Wilk and M. Nusair, Can. J. Phys. 58, 1200 (1980).

9. P. E. Blochl, O. Jepsen and O. K. Andersen, Phys. Rev. B49, 16223 (1994).

10. K. A. Mills, R. F. Davis, S. D. Kevan, G. Thornton and D. A. Shirley, Phys. Rev. B22, 581 (1980).

11. J. G. Nelson, W. J. Gignac, S. Kim, J. R. Lince and R. S. Williams, Phys. Rev. B31, 3469 (1985).

12. T. Grandke, L. Ley and M. Cardona, Phys. Rev. B18, 3847 (1978).

13. S. Kim, J. G. Nelson and R. S. Williams, Phys. Rev. B31, 3460 (1985).

14. S. Kim, L.-S. Hsu and R. S. Williams, Phys. Rev. B36, 3099 (1987).

15. J. A. Rayne, Phys. Lett. 7, 114 (1963).

16. L.-S. Hsu, Phys. Lett. A184, 476 (1994).

17. W. L. McMillan, Phys. Rev. 167, 331 (1968).

18. J. H. Wernick, A. Menth, T. H. Geballe, G. Hull and J. P. Maita, J. Phys. Chem. Solids 30, 1949 (1969).

19. J.-J. Yeh and I. Lindau, At. Data Nucl. Data Tables 32, 1 (1985).

20. D. A. Shirley, Phys. Rev. B5, 4709 (1972).

21. G. Apai, P. S. Wehner, J. Stohr, R. S. Williams and D. A. Shirley, Solid State Commun. 20, 1141 (1976).

22. G. Rossi, I. Lindau, L. Braicovich and I. Abbati, Phys. Rev. B28, 3031 (1983). 
Copyright $\odot 2002$ EBSCO Publishing 